\title{
Nanoscale oxide growth on Al single crystals at low temperatures: Variable charge molecular dynamics simulations
}

\author{
A. Hasnaoui, ${ }^{1}$ O. Politano, ${ }^{1, *}$ J. M. Salazar, ${ }^{1}$ and G. Aral $^{2}$ \\ ${ }^{1}$ Universite de Bourgogne, UMR-5613 CNRS, Faculte des sciences Mirande, 9 Avenue Alain Savary 21078, Dijon Cedex, France \\ ${ }^{2}$ Department of Physics, Izmir Institute of Technology, Gulbahce Campus, TR-35437, Urla, Izmir, Turkey
}

(Received 11 May 2005; revised manuscript received 27 July 2005; published 24 January 2006)

\begin{abstract}
We investigate the oxidation of aluminum low-index surfaces [(100), (110), and (111)] at low temperatures (300-600 K) and three different gas pressure values. We use molecular dynamics (MD) simulations with dynamic charge transfer between atoms where the interaction between atoms is described by the Es+ potential composed of the embedded atom method (EAM) potential and an electrostatic contribution. In the considered temperature range and under different gas pressure conditions, the growth kinetics follow a direct logarithmic law where the oxide thickness is limited to a value of $\sim 3 \mathrm{~nm}$. The fitted curves allow us to determine the temperature and the pressure dependencies of the parameters involved in the growth law. During the adsorption stage, we observe a rotation of the oxygen pair as a precursor process to its dissociation. In most cases, the rotation aligns the molecule vertically to the $\mathrm{Al}$ surface. The separation distance after dissociation ranges from 3 to $9 \AA$. Atomistic observations revealed that the oxide presents a dominant tetrahedral $\left(\mathrm{AlO}_{4}\right)$ environment in the inner layer and mixed tetrahedral and octahedral $\left(\mathrm{AlO}_{6}\right)$ environments in the outer oxide region when the oxide thickness reaches values beyond $\sim 2 \mathrm{~nm}$.
\end{abstract}

DOI: 10.1103/PhysRevB.73.035427

PACS number(s): 81.15.Aa, 81.65.Mq, 82.20.Wt

\section{INTRODUCTION}

Oxidation of metal and metal alloy surfaces are of considerable intrinsic interest $\mathrm{t}^{1,2}$ and are also important for numerous applications ranging from microelectronics, and heterogeneous catalysis to protection against wear and corrosion. ${ }^{3}$ Oxide passive films formed on aluminum and aluminum alloys in air protect the surface against further oxidation and corrosion. ${ }^{4-6}$ This passivating effect is responsible for their successful use as engineering materials. They are also used in various types of microelectronic devices. ${ }^{7-12}$ For example, aluminum oxide is a promising candidate for alternative gate dielectrics in the nanoscale CMOS technology, because its dielectric constant is twice higher than $\mathrm{SiO}_{2}$, while the band gaps are comparable. ${ }^{12}$ The discovery of the formation of ultra-thin ordered $\mathrm{Al}_{2} \mathrm{O}_{3}$ layers on single-crystal surfaces, which may also serve as model catalyst support surfaces, ${ }^{13}$ has led to an interest in the oxidation process of transition metal aluminides. For all these applications, there is a need to gather information on the composition, the microstructure and the limiting thickness of the oxide films. ${ }^{7,9}$

A large amount of experimental and theoretical works have been focused on understanding the processes involved in the oxidation of aluminum. Several authors have studied the mechanism and the kinetics of the growth of aluminum oxides. ${ }^{14-16}$ Other works investigated the reaction of $\mathrm{O}_{2}$ molecules with aluminum surfaces ${ }^{17,18}$ and the initial transient low mobility of oxygen atoms. ${ }^{19}$ Although many experimental studies characterized the formation mechanism and the microstructure of these oxide films, ${ }^{20-23}$ many questions concerning the atomistic details are still open.

Previous theoretical approaches were mostly based on $a b$ initio approaches and density-functional theory (DFT). These works focused on the first stages of the $\mathrm{O}_{2}$ molecule reaction with the $\mathrm{Al}$ surfaces,${ }^{24-27}$ the dissociation process, ${ }^{28,29}$ and the stability of adsorption sites. ${ }^{30}$ However, the dynamic behavior of the growth process cannot be studied by such approaches which do not include thermal vibrations and are confined to very small system sizes. As a consequence, these results are restricted, for instance, to the stability of the adsorption sites (fcc and hcp hollows) at the initial stage of oxidation. The temperature effect may alter the stability of these sites and/or destroy them completely.

Molecular dynamics (MD) simulations of aluminum oxidation have proven to be a valuable complementary tool for the investigation of both the oxide structure and the atomiclevel details of the growth mechanism. ${ }^{31-33}$ Ogata and Campbell ${ }^{31}$ and Campbell et al..$^{32}$ have done the first variable charge MD simulations of oxidation of $\mathrm{Al}$ spherical nanoclusters where the thickness of the amorphous oxide film has been found to saturate at 3 to $4 \mathrm{~nm}$. Gutierrez and Johansson ${ }^{34}$ have investigated the structural properties of amorphous alumina $\left(\mathrm{Al}_{2} \mathrm{O}_{3}\right)$ using $\mathrm{MD}$ simulations with fixed atomic charges. They found that the coordination number of the elementary unit of the oxide increases as its density increases. In a previous paper, ${ }^{33}$ we have investigated the oxidation of aluminum single crystal slabs using variable charge MD simulations at room temperature under a gas pressure of $9.8 \times 10^{+5} \mathrm{~Pa}$. Neither the temperature nor the pressure effects were investigated. We found that the growth kinetic is independent of the crystallographic orientations. We observed also the presence of an important density of mobile voids in the obtained amorphous structure of the oxide.

In the case where voids exist in the amorphous oxide layer, the growth kinetics is usually described by a directlogarithmic law [Eq. (6) below]. To our knowledge, neither experimental nor theoretical studies have treated the effect of the oxidation temperature and the gas pressure on this law. Although this law includes the effect of the temperature 


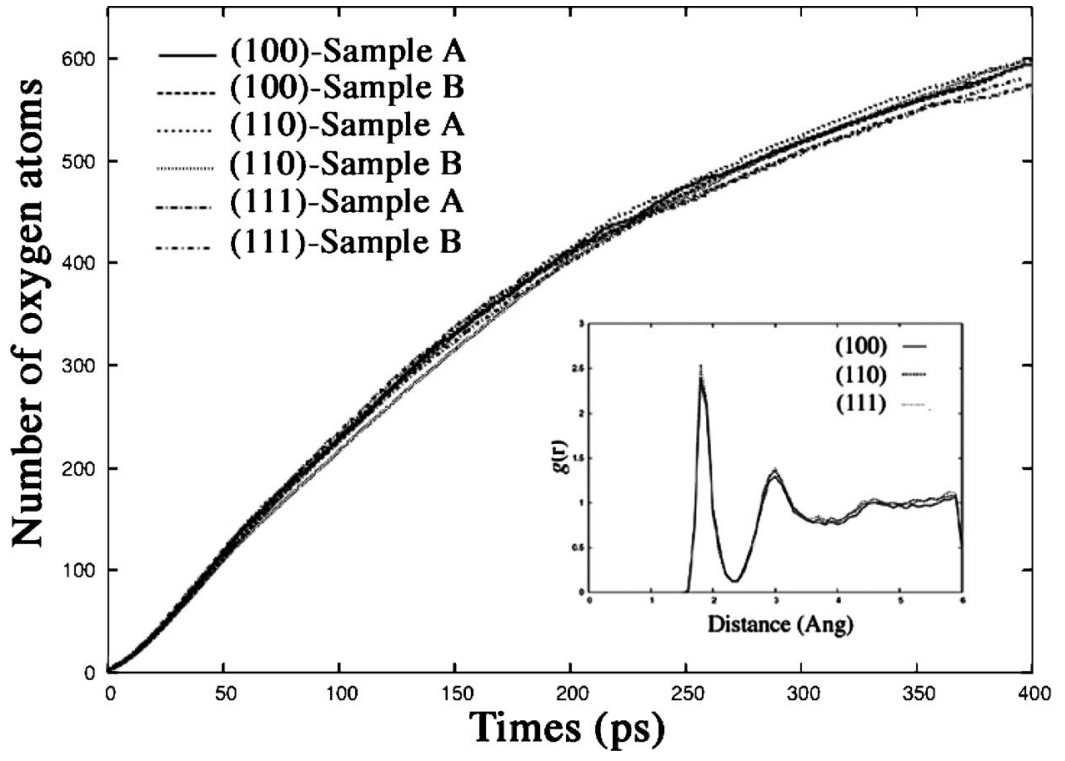

FIG. 1. Oxidation kinetic curves for the (100), (110), and (111) surface orientations using samples $\mathrm{A}$ and $\mathrm{B}$ at $300 \mathrm{~K}$ and $9.8 \times 10^{+5} \mathrm{~Pa}$. These curves represent the total uptake (as a number) of oxygen atoms versus exposure time; the curves for sample B were divided by four to allow comparison. The inset shows the total pair distribution function of the oxide film for the three faces in the case of sample B. through one parameter, it does not explain clearly how the temperature will affect the whole law. That is why in the present work, we investigate the effect of the temperature and the oxygen pressure on the oxidation kinetics. We also used larger samples compared to the ones used in Ref. 33 to emphasize the size effect on the results. Moreover, we shed light on some aspects of how the different terms in the direct-logarithmic law vary versus the oxidation temperature and the gas pressure. Then, we present atomic scale observations at room temperature corresponding to the different oxidation stages. These stages cover the oxygen molecule dissociation, the formation of the limiting thickness and include intermediate oxygen coverage stages. In the last section, we discuss our results and compare them to existing experimental and theoretical works when it is possible.

\section{COMPUTATIONAL METHOD}

The MD technique used here is based on an interaction potential developed by Streitz and Mintmire ${ }^{35}$ who proposed that the total energy of the crystal is divided into an electrostatic contribution (ES) and a nonelectrostatic energy (EAM potential originally proposed by Finnis-Sinclair $\left.{ }^{36}\right)$. A cutoff radius $\left(r_{c}\right)$ of $\sim 6 \AA$ is used for both the EAM interaction potential and the real-space contribution of the electrostatic potential. To compute the Coulomb interaction we use the classical three-dimensional Ewald summation technique, ${ }^{37}$ which is adapted to our two-dimensional slab geometry by adding two vacuum slabs on each side of the fcc aluminum substrate. ${ }^{38,39}$ The samples are thermalized by increasing the temperature in steps of $20 \mathrm{~K}$ from 0 to $300 \mathrm{~K}$ with successive runs of 1000 isokinetic MD steps by ignoring the dynamic charge transfer between atoms and by using a Berendsen thermostat. ${ }^{40}$ During this equilibration runs the surfaces are allowed to freely relax. Then, we perform a 1 ps NVT run with charge transfer to obtain the final $300 \mathrm{~K}$ relaxed system. After this equilibration stage, we introduce $\mathrm{O}_{2}$ molecules in the vacuum at $x=1.5 r_{c}$ from the aluminum surface and with random $y$ and $z$ positions. The $\mathrm{O}_{2}$ molecules are introduced with velocities randomly chosen from a Maxwell-Boltzmann distribution corresponding to the required temperature. Nevertheless, their initial component along the $x$ direction (the oxidation direction) is biased to have a molecule motion towards the substrate surface. Moreover, reflecting boundary conditions in the $x$ direction are imposed to those molecules that may reach the simulation box limit. Simulations with constant gas pressure are performed by introducing new $\mathrm{O}_{2}$ molecules each time the gas pressure is below the required one. A multiple time steps method, with $\delta t=1 \mathrm{fs}$ for the short-range forces and $\Delta t$ $=5 \mathrm{fs}$, for the long-range forces is used to integrate the equations of motion. In the following, one MD step will refer to the short-range forces time step, i.e., to $\delta t$. Given that the charge relaxation procedure, using the conjugate gradient method, is very time consuming, we decided to update the atomic charges every 100th MD step (every $0.1 \mathrm{ps}$ ). This is done by minimizing the electrostatic energy subjected to the constraint of the electroneutrality principle. The influence of a more frequent update (every $0.01 \mathrm{ps}$ ) was investigated using a simple example that consists of the reaction of a single $\mathrm{O}_{2}$ molecule with the $\mathrm{Al}$ surface of sample A. The results did not show any significant difference with those obtained with an update every $0.1 \mathrm{ps}$. All simulations are stopped when $\mathrm{AlO}_{4}$ fragments eject from the sample surface to the gas phase due to a local melting of the surface. ${ }^{32}$ For more details on the simulation technique see Ref. 33 .

\section{OXIDE GROWTH KINETICS}

Size effect. In this section we would like to elucidate the size effect, if any, on the oxidation of three Al single crystals [(100), (110), and (111)] at $300 \mathrm{~K}$. The simulated samples contain 1000 (sample A) or 4000 (sample B) atoms and we used a gas pressure value of $p_{0}=9.8 \times 10^{+5} \mathrm{~Pa}$. The oxidized surface area of sample A has a dimension of $(20 \times 20) \AA^{2}$ and that of sample $B$ is of $(40 \times 40) \AA^{2}$, with an aluminum slab thickness of $40 \AA$ for both samples. Figure 1 presents the kinetic curves of the three orientations of samples A and B as 


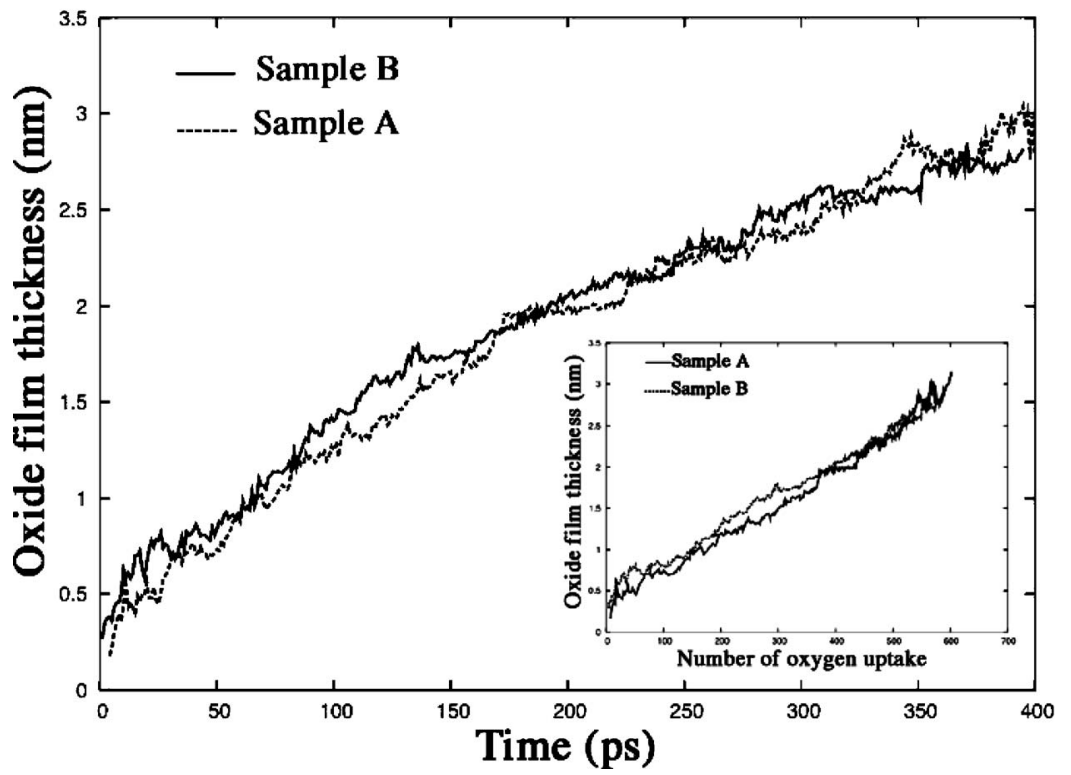

FIG. 2. The oxide film thickness versus exposure time for the (111) surface of samples $A$ and $\mathrm{B}$ at $300 \mathrm{~K}$ and $9.8 \times 10^{+5} \mathrm{~Pa}$. The inset presents the relationship between the oxide film thickness and the total number of oxygen atoms. the number of oxygen atoms deposited on the surface versus oxidation time (exposure time). The number of oxygen atoms uptake in the case of sample B have been divided by a factor of 4 to take account for the larger size of the oxidized area. This figure shows first that there is no noticeable effect of the crystallographic orientation on the oxidation of both samples A and B. Moreover, we did not observe any size effect on the oxide growth kinetics and its structure for the three surface orientations. We have also analyzed the oxide film structure, in the case of sample $B$, in terms of pair distribution functions (PDF's). In the inset of Fig. 1, we observe that these PDF's present similar curves and have a dominant peak at $\sim 1.8 \AA$ for the three orientations. These results for sample B will not be developed here since they do not exhibit significant difference to those obtained using sample A for which a more detailed study has been presented in Ref. 33. Figure 2 presents the oxide film thickness versus oxidation time for the (111) orientation of samples A and B. The oxide film thickness was computed with the technique explained in Ref. 33. In Fig. 2 we see that the thickness tends to a limiting value of $\sim 3 \mathrm{~nm}$. A similar result is also obtained for the two other orientations, which showed the same kinetic curves as exhibited by Fig. 1. The inset in Fig. 2 shows how the oxide thickness $X(t)$ varies versus the number of oxygen atoms uptake $N(t)$. After a transition regime ( $\sim 300$ oxygen atoms), we obtain a linearity of the form $X(t)=0.005 N(t)$ [see Eq. (5b)], which corresponds to a partial density of oxygen atoms in the oxide of $0.05 \AA^{-3}$. This value is independent of the considered pressure/temperature and agrees well with the directly computed density reported in Ref. 33.

In Fig. 3 we show a section of atoms of sample B, viewed in the oxidation direction, where small light gray dots represent $\mathrm{Al}$ atoms and black dots represent $\mathrm{O}$ atoms. In this figure we observe a region without deposited oxygen atoms on the top of the (110) surface [Fig. 3(a)]. This hole, of one monolayer depth, subsists until an oxidation time of $75 \mathrm{ps}$, whereas for the two other faces some holes also appeared and are filled with oxide earlier [see Fig. 3(b)]. We believe that this particular feature observed on the (110) surface of sample B is due to statistical fluctuations since it was not observed in the case of the (110) surface of sample A. Moreover, we think that the random choice of the $\mathrm{O}_{2}$ molecule initial positions in the gas might be responsible for this behavior.

We have also simulated an aluminum bicrystal with a $30^{\circ}$ $\langle 111\rangle$ tilt grain boundary. The two crystals were oriented along a (111) face. The simulation did not show any difference in the kinetic curves between the bicrystal and the single (111) crystal. Moreover, no oxygen migration was observed along this $\{111\}$-grain boundary even when we increased the system temperature to $600 \mathrm{~K}$. Note however that this result, under the present conditions, needs to be confirmed by more detailed studies.

Effect of the exposure temperature. Due to the absence of size and crystallographic orientation effects on the oxidation kinetics, we will use, in this section, only the (111) surface orientation of sample A. We have performed several simulations by varying the temperature from 300 to $600 \mathrm{~K}$ for two values of the oxygen pressure $p_{0}\left(=9.8 \times 10^{+5} \mathrm{~Pa}\right)$ and $3 p_{0}$. The resulting curves are plotted in Figs. 4(a) and 4(b) for $p_{0}$ and $3 p_{0}$, respectively. We see, that the growth kinetics are faster when increasing the oxidation temperature, for the two gas pressure values, as expected. This effect has been observed experimentally by Starodub et al. ${ }^{16}$ (at $\sim 4 \times 10^{-2} \mathrm{~Pa}$ ) and Jeurgens et al. ${ }^{20,21}$ (at $1.33 \times 10^{-4} \mathrm{~Pa}$ ) for temperatures below $673 \mathrm{~K}$. Notice that the latter authors observed an inversion of this behavior above $673 \mathrm{~K}$ for long exposure times that are not accessible to the MD technique, which they attributed to a reduction of the sticking coefficient of oxygen on the metal surface with increasing temperature.

For the studied temperature-gas-pressure regime, the oxidation kinetic curves show an initial stage of fast oxide-film growth, followed by a low growth rate stage. The increase in the gas pressure from $p_{0}$ to $3 p_{0}$ shifts the curves to shorter times leading to faster kinetics. This is explained by the supply of oxygen atoms at the $\mathrm{Al} / \mathrm{O}$ interface. The curves in Fig. 4(b) show that the growth becomes very slow beyond an 

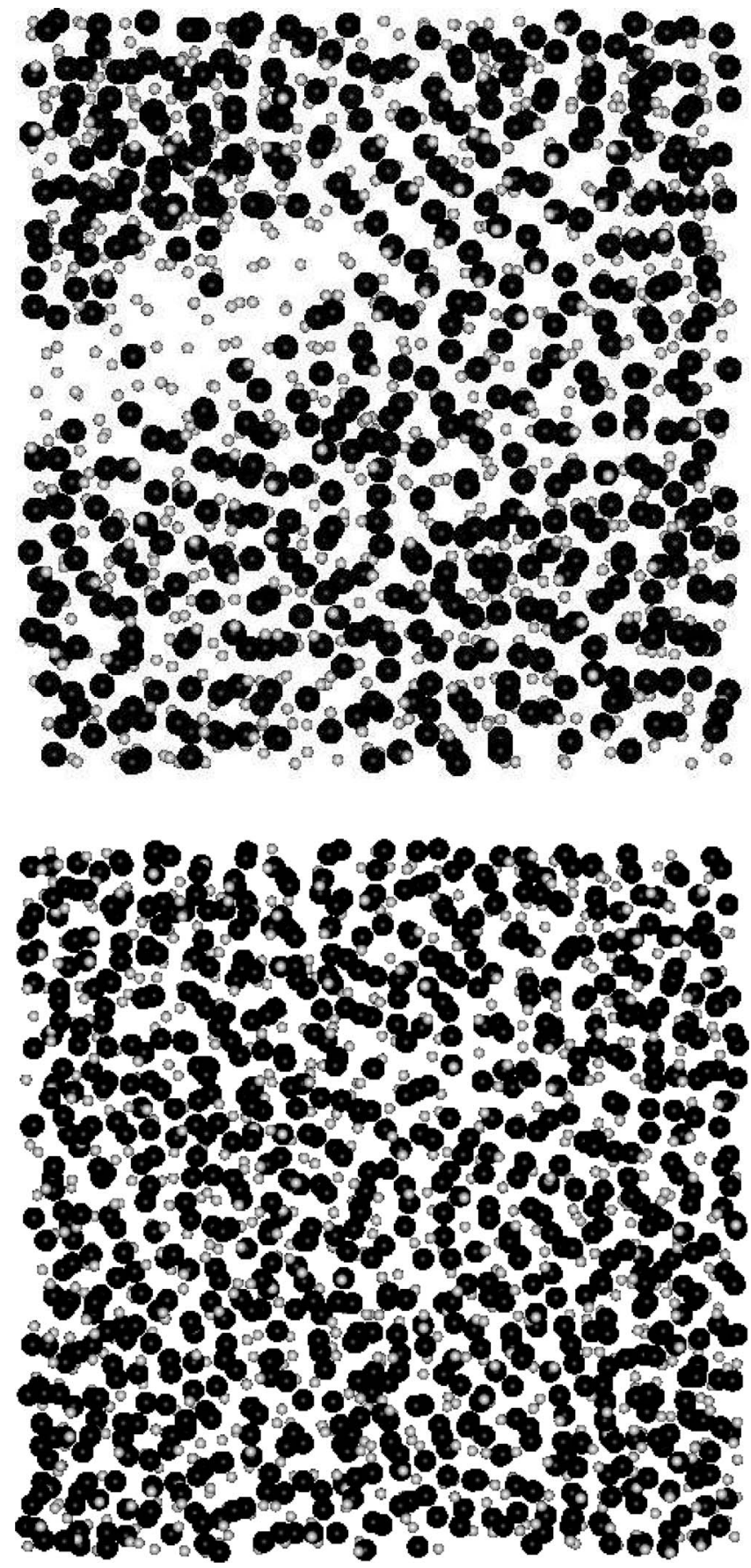

FIG. 3. A top-view of snapshots of the oxide film taken at $75 \mathrm{ps}$ exposure time. (a) Shows the existence of a hole in the oxide formed on the (110) surface and (b) shows the homogeneous oxide structure formed on the (111) surface. For the (100) surface we observed a homogeneous oxide after an exposure time of $75 \mathrm{ps}$ similar to what is shown in Fig. 3(b).

exposure time $t_{0}$. This time $t_{0}$ decreases when the temperature increases. This figure shows also a limiting value of the number of oxygen atoms (uptake), which is similar for the temperature range between 300 and $600 \mathrm{~K}$. If we extrapolate these curves to longer times, we would obtain a number of oxygen atoms that should correspond to a limiting thickness of $\sim 3 \mathrm{~nm}$. This is in agreement with the general tendency of aluminum oxidation kinetics at low temperatures $(<600 \mathrm{~K}),{ }^{14,16,32}$ where a limiting regime is observed around an oxide thickness of 1-4 nm.

For the temperature/pressure regime studied here, the oxidation kinetic curves follow a direct-logarithmic growth beyond a transient regime. Figure 5 shows, for the (111) orientation of sample A, an example of the fits of the kinetic curves at $300 \mathrm{~K}$ where the number of oxygen uptake is fitted to a direct logarithmic function of exposure time $[\alpha \ln (t)$ $+\beta]$. In this figure, the fit is performed over exposure times greater than $90 \mathrm{ps}$. This direct logarithmic growth mode is usually related to a mechanism where the oxide develops by ionic movement via extended defects, namely voids in the present case.

According to the theory of oxidation kinetics in ultra-thin films, the general expression for a potential to be overcome for a field-assisted migration of an ion between two adjacent sites is given by ${ }^{41}$

$$
W=W_{0}-1 / 2 q a E+\lambda X,
$$

where $W_{0}$ is the intrinsic barrier for ionic jumps between two positions in the oxide, $q$ the ionic charge, $2 a$ the jump length, $\lambda$ is a term that depends of the oxide structure, and $X$ is the oxide thickness. The right hand second term describes the lowering of the energy barrier by an electric field $E$ across the oxide, and the third term expresses the oxide structural change as the film thickness develops. A possible microscopic mechanism for ion movement is via extended defects in the oxide layer, such as structural channels (voids in our case). When the film grows, these easy pathways become blocked by the formation of oxide within them. The activation energy is then larger for thicker films and its change is proportional to the oxide thickness. The growth rate equation, in this case, is given by ${ }^{41}$

$$
\frac{d X}{d t}=c \exp \left(-\frac{W_{0}-1 / 2 q a E+\lambda X}{k_{B} T}\right)
$$

where $k_{\mathrm{B}}$ is the Boltzman constant and $c$ is a constant. The solution of this equation is a direct logarithmic function ${ }^{16}$

$$
X(t)=\left(k_{B} T / \lambda\right) \ln [1+\xi(T) t],
$$

where $\xi(T)$ is defined by

$$
\xi(T)=\left(\frac{\lambda}{k_{B} T}\right) \cdot c \cdot \exp \left[-\frac{W_{0}-1 / 2 q a E}{k_{B} T}\right] .
$$

Equation (3) reduces, for large times $[\xi(T) t>1]$, to

$$
X(t)=\frac{k_{B} T}{\lambda} \ln [\xi(T)]+\frac{k_{B} T}{\lambda} \ln (t),
$$

which corresponds to the linear dependence of the oxide thickness $X(t)$ on $\ln (t)$. 

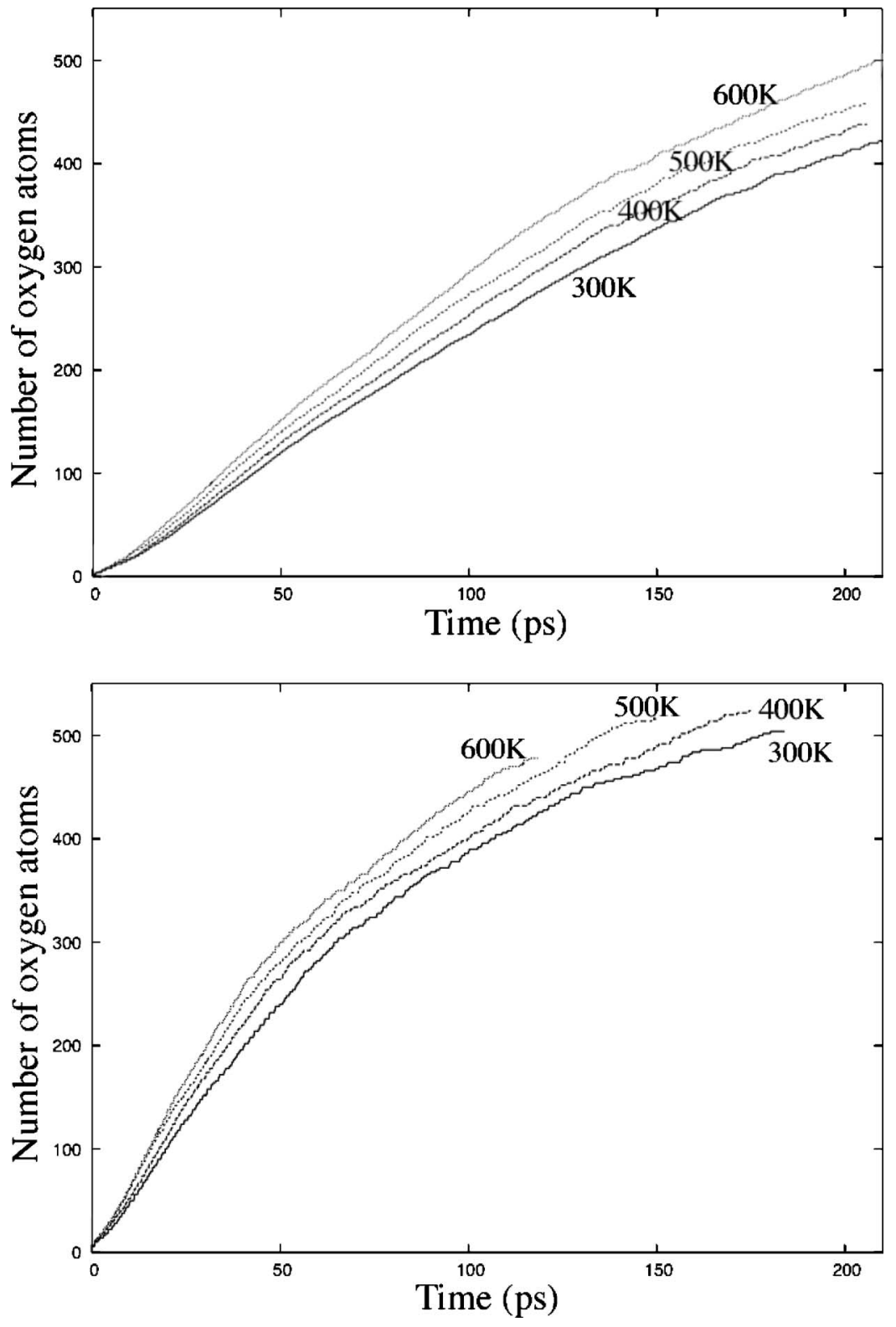

FIG. 4. Oxidation kinetic curves of the (111) surface of sample A for a temperature ranging from 300 to $600 \mathrm{~K}$. These curves represent the total uptake (as a number) of oxygen atoms versus exposure time. The oxidation is performed at a gas pressure of (a) $p_{0}\left(9.8 \times 10^{+5} \mathrm{~Pa}\right)$ and (b) $3 p_{0}$.
In this work, due to the errors that may occur when computing the oxide thickness we prefer to plot and fit the oxygen uptake $N(t)$ as a number of atoms added to the Al substrate. This number $N(t)$ is proportional to the thickness $X(t)$ in an homogeneous oxide, and their ratio is a function of the exposed surface area of the oxidized sample $(A)$ and the density of oxygen atoms in the oxide $(\rho)$ :

$$
\frac{N(t)}{X(t)}=\rho A .
$$

This relationship is justified in our simulation results since the obtained amorphous oxide film is homogeneous and does not show any roughness features before reaching the limiting regime.

Equation (5) can then be rewritten as

$$
N(t)=\alpha \ln (t)+\beta,
$$

where the parameters $\alpha$ and $\beta$ are defined by

$$
\alpha=\frac{k^{\prime} T}{\lambda} \text { and } \beta=\frac{k^{\prime} T}{\lambda} \ln [\xi(t)]
$$

where $k^{\prime}=k_{\mathrm{B}} A \rho$. The density $\rho$ being constant beyond a certain time (typically 100 ps at $300 \mathrm{~K}$ ) and does not vary with the temperature within statistical fluctuations. In the previous subsection the product $\rho A$ has been found to be equal to $200 \mathrm{~nm}^{-1}$, which corresponds to the inverse of the slope of the inset in Fig. 2.

We obtain $\alpha$ and $\beta$ from fitting the kinetic curves in Fig. 4 on Eq. (6). From these two parameters we deduce the values of the structure term $\lambda$ (in $k^{\prime}$ unit) and the term $\xi(T)$ $\times\left(k_{\mathrm{B}} T / \lambda\right)$ using Eqs. (4) and (7). The results are plotted in Fig. 6(a) for the variation of the structure term $\lambda$ versus the temperature $T$ and in Fig. 6(b) for the variation of the term 


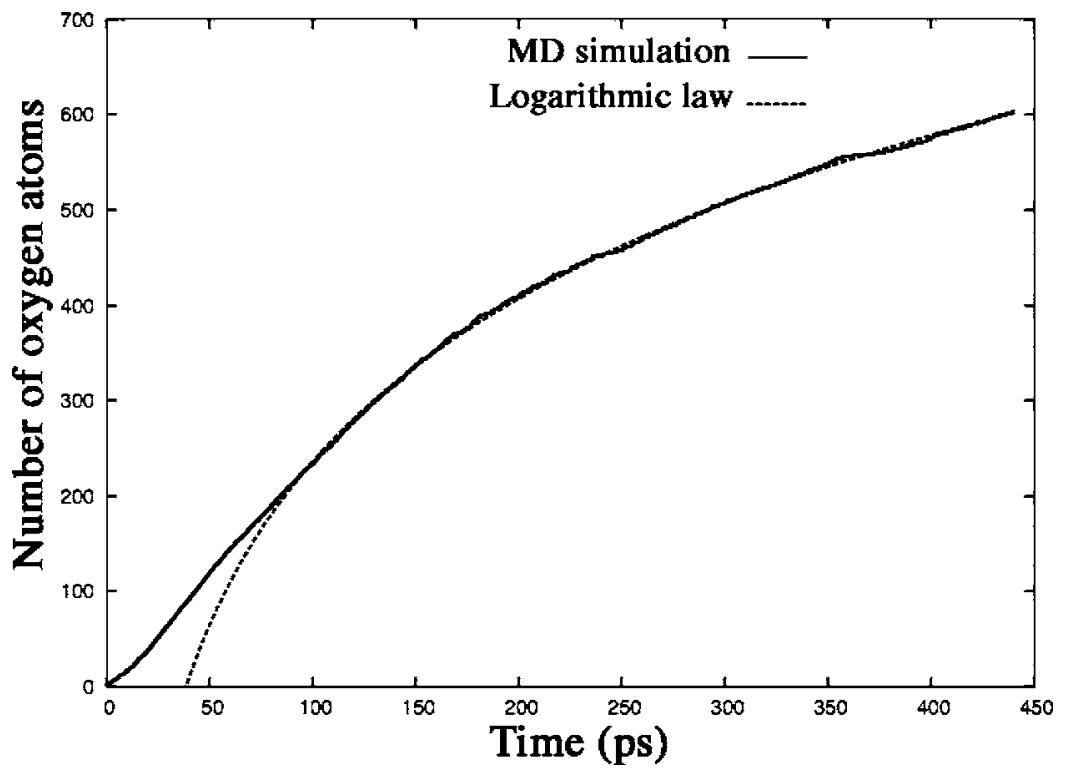

FIG. 5. Typical illustration of the fits of the kinetic curves on a direct-logarithmic law using an (111) orientation of sample A oxidized at $300 \mathrm{~K}$ and $9.8 \times 10^{+5} \mathrm{~Pa}$. The fit has been performed on exposure times greater than $90 \mathrm{ps}$.

$\xi(T)\left(k^{\prime} T / \lambda\right)$ as a function of $1 / T$. We see from this figure that the structure term $\lambda$ (in $k^{\prime}$ units) is a linear function of temperature and can be described by the following relationships for the two gas pressure values $p_{0}$ and $3 p_{0}$, respectively,

$$
\begin{aligned}
& \lambda=3.2710^{-3} T+0.246, \\
& \lambda=4.5010^{-3} T+0.159 .
\end{aligned}
$$

The term $\xi(T)\left(k_{\mathrm{B}} T / \lambda\right)$, shown in Fig. 6(b), presents the Arrhenius dependence on the temperature, as expected from Eq. (4). The fits of these curves allow writing relationships (10) and (11) for the two gas-pressure values $p_{0}$ and $3 p_{0}$, respectively,

$$
\begin{aligned}
& c \exp \left(-\frac{W_{0}-1 / 2 q a E}{k_{B} T}\right)=\exp \left(-147.5 \frac{1}{T}-4.60\right), \\
& c \exp \left(\frac{W_{0}-1 / 2 q a E}{k_{B} T}\right)=\exp \left(-176.6 \frac{1}{T}-3.75\right) .
\end{aligned}
$$

These Arrhenius plots lead to an estimation of the term $W$ $=W_{0}-1 / 2 q a E$ to $(0.013 \pm 0.01) \mathrm{eV}$ and $(0.015 \pm 0.02) \mathrm{eV}$ for $p_{0}$ and $3 p_{0}$, respectively. In an amorphous oxide structure, it is difficult to estimate the jump distance $a$ because we don't have access to the value of the electric field $E$. At this point, we are not able to calculate accurately the intrinsic energy barrier. However, it can be estimated using published values of the electric field $\left(\sim 7 \times 10^{+6} \mathrm{~V} \mathrm{~cm}^{-1}\right) .{ }^{14,16}$ Considering the electronic charge of oxygen $q \sim 1.8 e$ and the oxygen jump distance as the O-O PDF first peak $(\sim 3 \AA)$, we computed the term $1 / 2 q a E$ to be $0.38 \mathrm{eV}$. The intrinsic energy barrier $W_{0}$ is then evaluated to be $0.39 \mathrm{eV}$, which is lower than the published values. Starodub et al. ${ }^{16}$ found $1.67 \mathrm{eV}$ with the inverse log law using thinner oxide films (e.g., $1.5 \mathrm{~nm}$ for $573 \mathrm{~K}$; whereas in this work we obtained at least $2.3 \mathrm{~nm}$ ). They assumed an oxygen charge $q=2 e$ and a jump distance $a=2.48 \AA$ and suggested that the growth mechanism occurs by oxygen migration via point defects (vacancies). Jeurgens et al. ${ }^{14}$ using the Cabrera Mott theory, found the energy barrier $2.5-2.6 \mathrm{eV}$ considering cation migration as the rate limiting process that is located at the metal/oxide interface. The difference on the value of the energy barrier we obtained, with respect to the previous authors, can be attributed to the fact that in our simulations (1) the oxygen pressure is many order of magnitudes higher than the experimental value, (2) the growth mechanism takes place by both $\mathrm{Al}$ and $\mathrm{O}$ migration, (3) the energy barrier is located in the oxide, and (4) its lower value also results from the presence of voids in the oxide, which accelerates the growth kinetics.

Effect of the oxygen pressure. Figure 7(a) illustrates the oxygen pressure effect on the oxidation kinetics at $300 \mathrm{~K}$. Kinetics are faster for larger oxygen pressure, as expected. Zheludkevich et al. $^{42}$ have seen the same effect for silver oxidation at $523 \mathrm{~K}$ by varying the value of the flux of atomic oxygen. The curves in Fig. 7(a) fit the direct-logarithmic law of Eq. (6) well. The fitted parameters allowed us to determine the structure factor $\lambda$ and the term $\xi(T)\left(k_{\mathrm{B}} T / \lambda\right)$, which we plotted in Fig. 7(b) as a function of oxygen pressure. The term $\xi(T)\left(k_{\mathrm{B}} T / \lambda\right)$ is scaled by a factor of 100 to allow its visualization within the graph scale. From this figure we are able to confirm that the two terms are sensitive to gas pressure and in consequence the function $\xi(T)$ should depend also on the oxygen pressure and not only on the exposure temperature. However, it is not clear how the terms used in Eq. (6) will vary with the gas pressure in another interval mainly if the gas pressure is many orders of magnitude lower than $p_{0}$, which is not accessible to the MD simulations.

\section{ATOMISTIC OBSERVATION AND GROWTH MECHANISM}

At the very early times (adsorption stage), we observed that when the oxygen molecule arrives on the aluminum surface, two aluminum atoms jump out of the surface. Looking at the top view we saw that the oxygen atoms arrive on both 

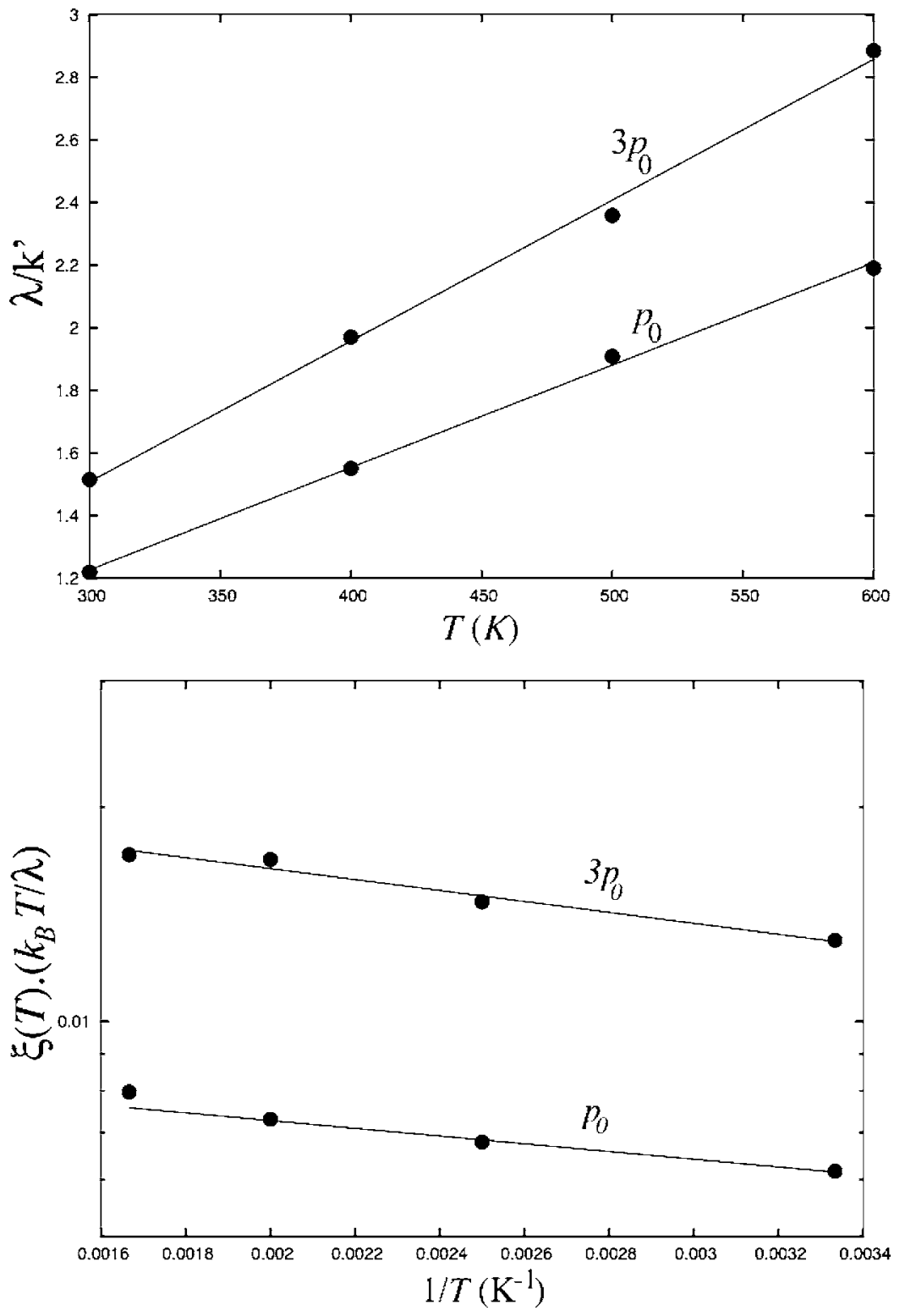

FIG. 6. Variation of the fit parameters, obtained from fitting curves in Figs. 4(a) and 4(b) on Eq. (6), with the temperature. (a) The structure term $\lambda$ (in $k^{\prime}$ units) versus $T$ and (b) The term $\xi(T)\left(k_{\mathrm{B}} T / \lambda\right)$ versus $T$ in an Arrhenius plot. fcc and hep hollow sites. The structure then relaxes such that oxygen atoms locate in tetrahedral sites (surrounded by four $\mathrm{Al}$ atoms). The formation of this structure unit requires aluminum atomic jumps both out of the surface (atoms numbered 1 to 4 in Fig. 8) and from the aluminum sublayer to interstitial-like sites (atoms numbered 5 and 6 in Fig. 8). The charges of oxygen atoms, in this configuration, are $-1.43 e$ and $-1.49 e$ (with $e$ is the magnitude of electronic charge). Those of surrounding $\mathrm{Al}$ atoms are $+0.86 e$ for the $\mathrm{Al}$ atoms that are a common neighbor to both oxygen atoms and vary between +0.45 and +0.56 for the other $\mathrm{Al}$ atoms. DFT calculations ${ }^{25}$ have shown that the charge on the adsorbed oxygen molecule is always well below two electrons.

We have investigated the dissociation of 12 oxygen molecules on a $(111)$ Al sample with $(100 \times 100) \AA^{2}$ exposed surface area and a thickness of $22 \AA$. The large exposed surface area has been chosen to prevent interaction between oxygen molecules, at least in the early stage of oxidation. We calculated the angle that each molecule makes with the normal to the oxidized surface. We also computed the evolution of the position of the center-of-mass of the molecule and the separation distance of the two oxygen atoms coming from the same molecule. When oxygen arrives on a clean Al surface, the general trend is that the pair rotates to have a vertical direction when its center of mass is about 2-3 $\AA$ above the Al surface [Fig. 9(a)]. When the molecule approaches closely the surface, it rotates again to make an angle with the vertical that is between $10^{\circ}$ and $60^{\circ}$. This is accompanied by a slight extension of the bond length. The separation distance between atoms of each molecule showed that the dissociation starts effectively when the center of mass of the molecule is located at a position that ranges between 0.8 and $1.5 \AA$ above the surface [Fig. 9(b)]. The rotation of the $\mathrm{O}_{2}$ molecule is a precursor to the dissociation. The separation distance immediately after the complete dissociation process ranges from $3 \AA$ to $5 \AA$, and due to atomic rearrangements and oxygen molecules interactions it may reach $9 \AA$, in some cases. This is in agreement with experimental results ${ }^{19}$ and DFT calculations, ${ }^{25}$ where the final distance after separation ranges between one and three times the $\mathrm{Al}$ interatomic spac- 

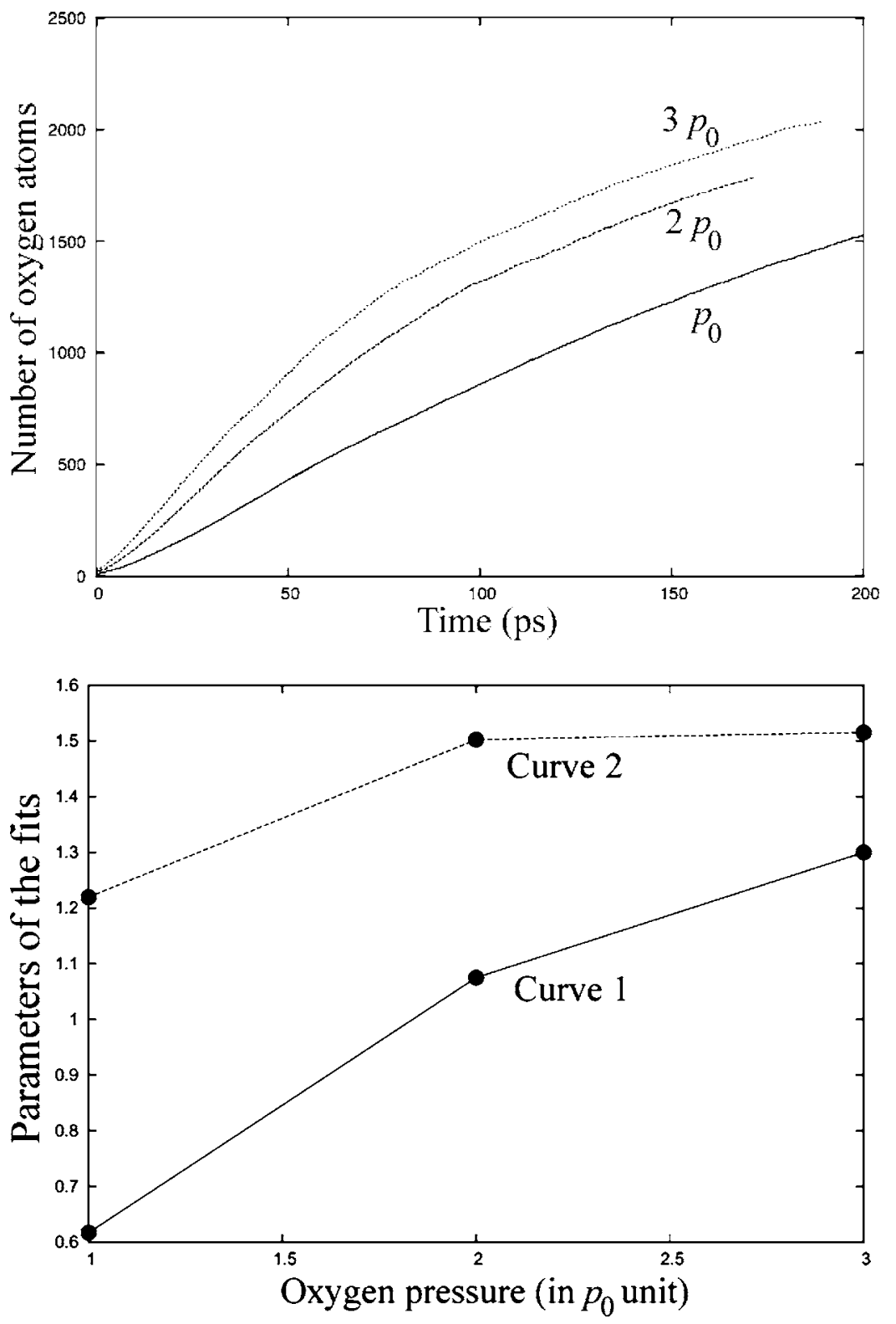

FIG. 7. (a) Oxide kinetic growth curves for the (111) surface of sample B at $300 \mathrm{~K}$ with different oxygen pressures (with $p_{0}=1.98$ $\times 10^{+5} \mathrm{~Pa}$ ). These curves represent the total uptake (as a number) of oxygen atoms versus exposure time. (b) Parameters obtained from the fits of the curves of (a) on the direct-logarithmic growth law [Eq. (6)]. Curve 1 corresponds to $\xi(T)$ $\left(k_{\mathrm{B}} T / \lambda\right)$ scaled by a factor of 100 and curve 2 corresponds to $\lambda$ (in $k^{\prime}$ units). The lines are drawn to guide the eye. ings. Note however, that we observed molecular dissociation only when both atoms are bound to the surface, and we did not observe abstractive adsorption process where one oxygen atom remains on the surface and the second emerges to the gas phase. This is due to the strong electrostatic attraction energy between $\mathrm{O}$ atoms and $\mathrm{Al}$ atoms from the substrate in the used interaction potential. After their dissociation, oxygen atoms have positions between $1 \AA$ above and $2 \AA$ below the surface plane. This indicates that independent oxygen molecules remain at the surface and that the deep penetration of oxygen is correlated to the presence of oxygen clusters.

Nevertheless, we have detected one case where the oxygen pair approached the Al surface in a horizontal orientation. In this case, the dissociation started at $\sim 2 \AA$ above the surface with a slight extension of the bond length. The complete dissociation occurred when the center-of-mass of the pair locates at $1 \AA$ above the surface and with a separation distance of $5 \AA$. The atoms of this particular dissociated molecule are located at $\sim 1 \AA$ above the surface. They did not penetrate beyond the surface geometrical plane during the dissociation process.

For coverages of less than 1 monolayer (1 ML), oxygen clusters (2D oxides) form by adsorption of supplementary oxygen atoms. Indeed, the new arriving oxygen atoms try to form locally a 2D-triangular network [Fig. 10(a)] with the pre-existing oxygen atoms. During this initial oxide formation we observed that oxygen atoms do not move considerably but only by minute displacements whereas aluminum atoms perform more important displacements to rearrange the structure around the oxygen atoms [black lines in Fig. $10(\mathrm{~b})]$. For the three aluminum faces, we observed that $\mathrm{Al}$ atoms rearrange to form a triangular network on the top layer. Figure 10(b) shows an example in the case of the (110) surface of this local configuration of $\mathrm{Al}$ atoms where oxygen atoms occupy the centers of the triangles. Black lines stand for $\mathrm{Al}$ atomic displacements between the time this snapshot is taken and the initial structure. Note that snapshots in Fig. 10 show a $2 \mathrm{D}$ representation taken at the $\mathrm{Al}$ surface, but in 


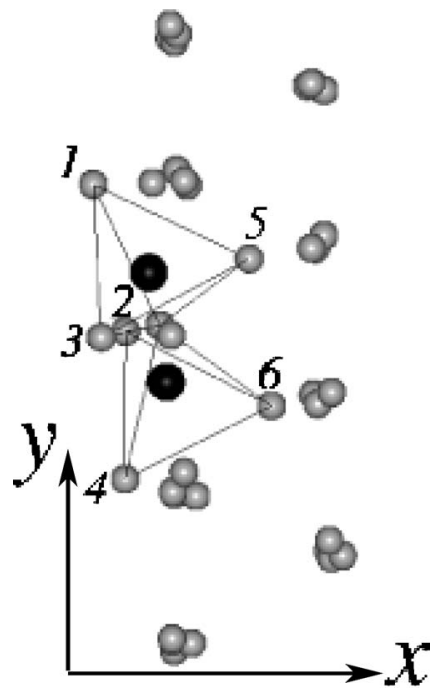

FIG. 8. A side view of the tetrahedral structure formation on the (111) Al-surface just after dissociation. Black balls represent oxygen atoms and gray balls represent $\mathrm{Al}$ atoms. The formation of this structure requires $\mathrm{Al}$ atomic jumps ( $\mathrm{Al}$ atoms numbered from 1 to 4) out of the Al surface planes. The (111) crystallographic axis is along the $x$ axis.

reality some slight distortion in the perpendicular direction may exist.

The (100) and (110) surfaces have shown an atomic rearrangement on the surface where the original structure transforms to a triangular network, whereas the (111) undergoes a slighter deformation to accommodate the oxygen incorporation. We should say that this rearrangement happens in regions where oxygen atoms are clustered whereas it keeps its original surface structure otherwise [see Fig. 10(a)]. Note also that the triangular network shown in Fig. 10(b) does not coincide with that of the (111) face of the $\mathrm{Al}$ fcc structure. Upon further exposure, more oxygen atoms arrive to the oxide surface and some oxygen atoms are then pushed to penetrate the next layer. During this time $\mathrm{Al}$ atoms leave the sublayer of the substrate and bond with oxygen atoms. This process leads to the transformation of the $2 \mathrm{D}$ cluster to a $3 \mathrm{D}$ oxide where oxygen atoms are located in tetrahedral sites, surrounded by $\mathrm{Al}$ atoms.

After this stage, the development of the oxide structure occurs in a layer-by-layer mode. Oxygen atoms penetrate to the next layer, attracting $\mathrm{Al}$ atoms that move from the $\mathrm{Al}-$ substrate layer across the metal-oxide interface to interstitiallike positions. This leads to the formation of a free volume at the interface. This free volume moves laterally on the interface layer (and never into the oxide) allowing a rearrangement of interfacial atoms forming a free space by which supplementary oxygen atoms can move forward.

We have analyzed the structure of the oxide in terms of $\mathrm{Al}-\mathrm{O}$ local atomic coordinations determined from the atomic cluster statistics and by using a sphere radius $R=1.2 r_{0}\left(r_{0}\right.$ is the value of the first peak of the PDF curves). We found that, during the early stages, atoms in the oxide have coordination 4 [dark gray balls in Fig. 11(a)] and then a tetrahedral environment. After further exposure time $(150 \mathrm{ps})$, we continue to observe this tetrahedral environment but mixed with a small amount of five-coordinated atoms. For an exposure time of $300 \mathrm{ps}$, we see that the structure of the oxide presents a dominant tetrahedral environment in the inner layer and a mixed tetrahedral and octahedral environments in the outer oxide region, as shown by the black balls (six-coordinated $\mathrm{Al}$ atoms in Fig. 11). Note that the tetrahedral environment is dominant for the three exposure times and that the amount of four-coordinated atoms decreases with time. We have observed that once oxygen and $\mathrm{Al}$ atoms form a tetrahedral network, oxygen atoms move towards the metal-oxide interface. Inversely aluminum atoms traverse this layer and form the left part of the oxide, whose structure is different from that close to the metal-oxide interface.

\section{DISCUSSION}

When cation migration prevails, the basic assumptions of the Cabrera-Mott theory ${ }^{43}$ are adopted and inverse logarithmic kinetics are obtained. The reaction rate in this case is not sensitive to oxygen pressure, but should vary with the crystallographic orientation. In our simulations, the results show that both anion, and to a lesser extent, cation migration occur (e.g., Fig. 9 in Ref. 33) for all the studied temperatures $(300$ to $600 \mathrm{~K}$ ). This movement through the amorphous oxide takes place via extended defects, namely voids in our case. This mechanism is accommodated by cation-lateral migration assisted by free volume diffusion along the interface. We did not observe a noticeable oxygen lateral diffusion, which is in agreement with the room temperature results of Brune et al. ${ }^{44}$ and Chakarova et al. ${ }^{45}$ The accommodation occurs by reorganization of the local structure to form tetrahedral structured oxide in the nearby metal/oxide interface. This leads to the creation of new local channels at the interface that allow the advance of oxygen atoms and the growth of the oxide film. We have also seen that there is no effect of the crystallographic orientation of the substrate (Fig. 2). Moreover, in Fig. 7 we observe that the increase of the gas pressure has the effect to speed up the kinetics of growth, which excludes the occurrence of the Cabrera-Mott ${ }^{43}$ mode. The present results are in agreement with a kinetic of growth that follows a direct-logarithmic law (Fig. 5) and are also supported by the presence of the voids in the oxide film. The parabolic law has also been tested and showed a deviation from the growth kinetic curve. This is also in agreement with the general behavior of very thin oxide films. ${ }^{16,41}$

The growth kinetics law given in Eqs. (6) and (7) does not take into account the temperature dependency of the structure factor. Moreover, this law does not include any gas pressure effect. Our analysis of the growth kinetic curves for different temperatures and different gas pressures allows us to determine the variation of the parameters involved in the direct-logarithmic law versus temperature and gas pressure. As shown in Fig. 6(a), the structure factor $\lambda$ is a linear function of the temperature and increases with the gas pressure. The function $\xi$ [see Eq. (4)] is in fact a product between a linear function (proportional to $\lambda$ ) and an Arrhenius function of the temperature as shown in Fig. 6(b). It depends also on the gas pressure as shown in Fig. 7(b) (curve 2). The abovementioned result allows us to get a deeper understanding of 

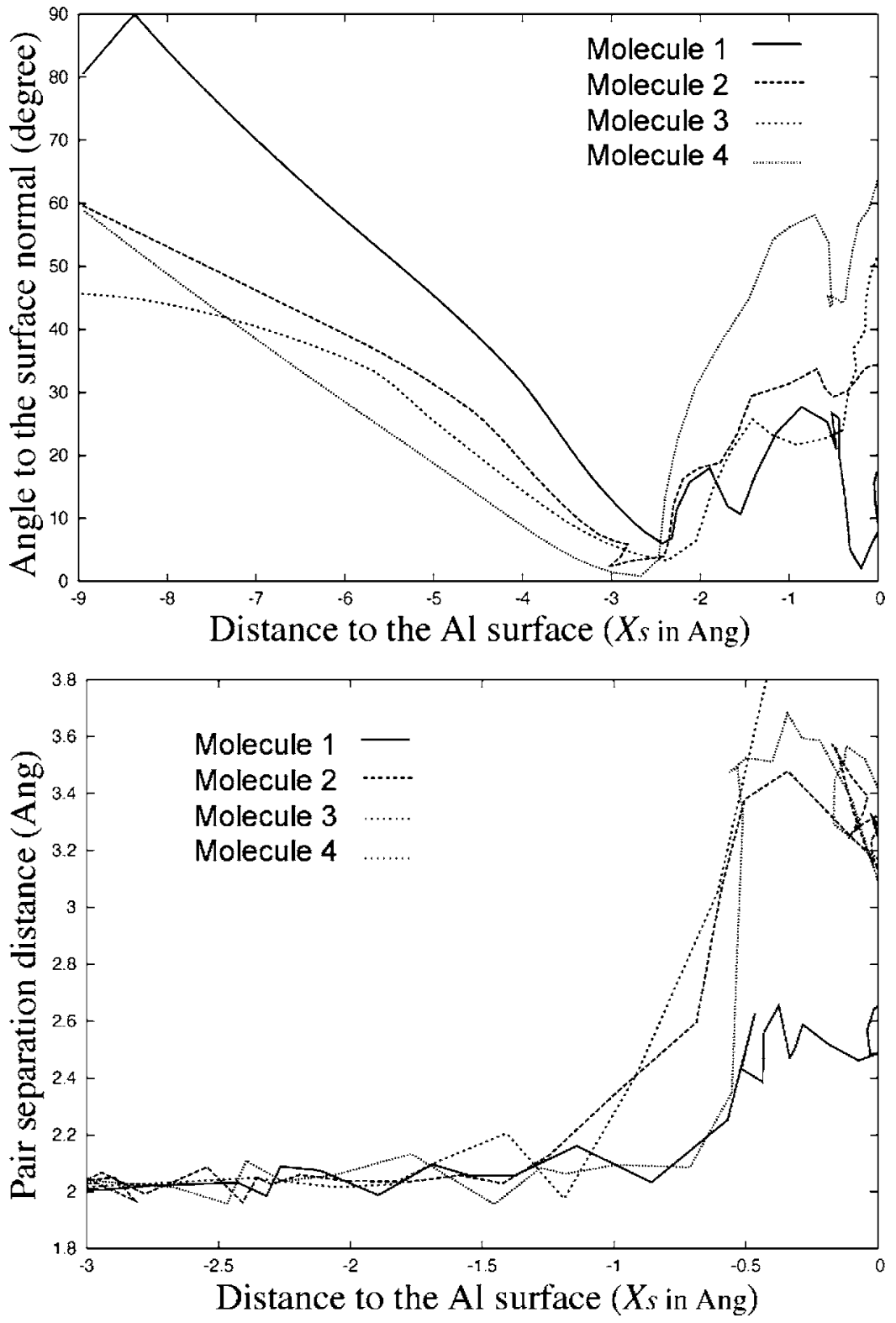

FIG. 9. (a) Evolution of the oxygen molecule/ pair angle with the surface normal plotted versus the position $\left(X_{s}\right)$ of the center-of-mass of the pair from the Al surface. (b) Separation distance of the two atoms of the oxygen molecule plotted versus $X_{s}$. Four typical examples of oxygen molecules are given. the growth kinetics of amorphous aluminum oxides, but at this stage we are not able to provide a more general equation including temperature and gas pressure dependencies. Note also that the used gas pressure values are many orders of magnitude higher than what is generally used experimentally, which is not accessible to MD technique. The use of such high values may have a non-negligible influence on the growth kinetic curve and the density of voids in the obtained oxide film.

Sasaki and Ohno, ${ }^{46}$ using DFT calculations, found that the bond length is elongated when the oxygen molecule is close to the Al surface. Our results show that dissociation can occur at a distance from the surface $X_{s}$ of about 0.8 to $1.5 \AA$. The distance between two oxygen atoms, originally from the same molecule, ranges after dissociation from 3 to $9 \AA$ with a distribution peak at $\sim 3.4 \AA$. This distribution has been taken from a $(111)$ sample with $(100 \times 100) \AA^{2}$-exposed surface to minimize the boundary effects on the O-O interaction. Wahnstrom and co-workers ${ }^{47,48}$ found, using MD simu- lations of the atomic motion in terms of the effective medium theory, that the $\mathrm{O}$ atom must have a kinetic energy of at least $9.5 \mathrm{eV}$ to account for the experimental results of Brune et $a l .{ }^{18}$ who found a random distribution of single $\mathrm{O}$ atoms that is supposed to result from a hyperthermal motion of $\mathrm{O}$ atoms. In our simulations, we calculated the kinetic energy of $\mathrm{O}$ atoms just after dissociation and found that this energy is generally lower than $1.3 \mathrm{eV}$ and does not fulfill the condition cited above. Our results support the observations in Refs. 47 and 48 and are in contradiction with the results of Brune et al. ${ }^{18}$ Note that the observed single oxygen atoms in Fig. 10 (a) have neighbors in the perpendicular direction and these neighbors are not always shown since they are out of the selected section of atoms that is very thin $(\sim 3-\AA$ thick $)$. Our results are corroborated by atomistic images showing a pair distribution of $\mathrm{O}$ atoms in the very initial stage and the formation of regions consisting of a triangular network [Fig. 10(b)]. 

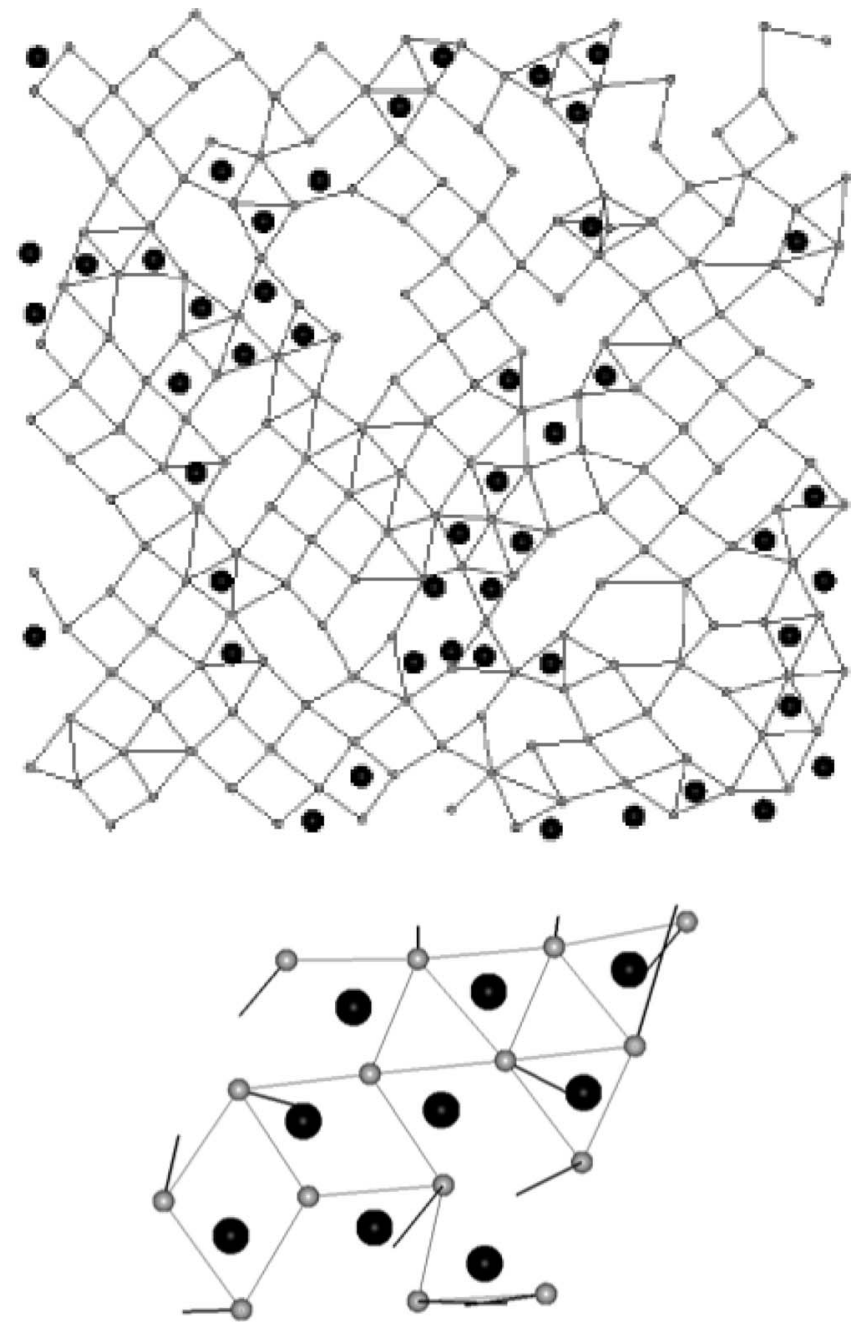

FIG. 10. (a) A top view of a 3 - $\AA$-thick section of atoms of the (100) Al-surface showing the triangular network locally formed for low oxygen coverage taken at 22 ps exposure time. (b) A top view of the structure of a section of atoms at the aluminum (110) surface after 26 ps of exposure. The black lines represent displacement of $\mathrm{Al}$ atoms during $26 \mathrm{ps}$. In (a) and (b) light gray color stands for aluminum atoms and darker one for oxygen atoms.

Kiejna et al. ${ }^{26}$ have performed DFT calculations to study the stability of the hcp and fcc hollow sites on the Al (111) surface for oxygen deposition. In our work, we have seen that oxygen atoms are adsorbed on both sites (fcc and hcp) and that due to the lack of robust statistics we cannot conclude which sites is more favorable. We think that due to thermal fluctuations (room temperature experiment) and to $\mathrm{Al}$ atomic rearrangement around oxygen atoms, the notion of fcc and hcp hollow sites loses its meaning, mainly at intermediate oxygen coverage when oxygen $2 \mathrm{D}$ clusters form.

The formation of an oxide compound generally involves exchange of atomic place. ${ }^{44}$ This process is usually assumed to occur via penetration of $\mathrm{O}$ atoms through the topmost layer of the $\mathrm{Al}$ substrate. However, as we saw in this work, this does not occur necessarily through the Al topmost layer. Rather it involves $\mathrm{Al}$ atom jumps (pulled out of the surface) from the topmost layer out of the surface and $\mathrm{Al}$ atom jumps from the subsurface layer to an interstitial-like position just
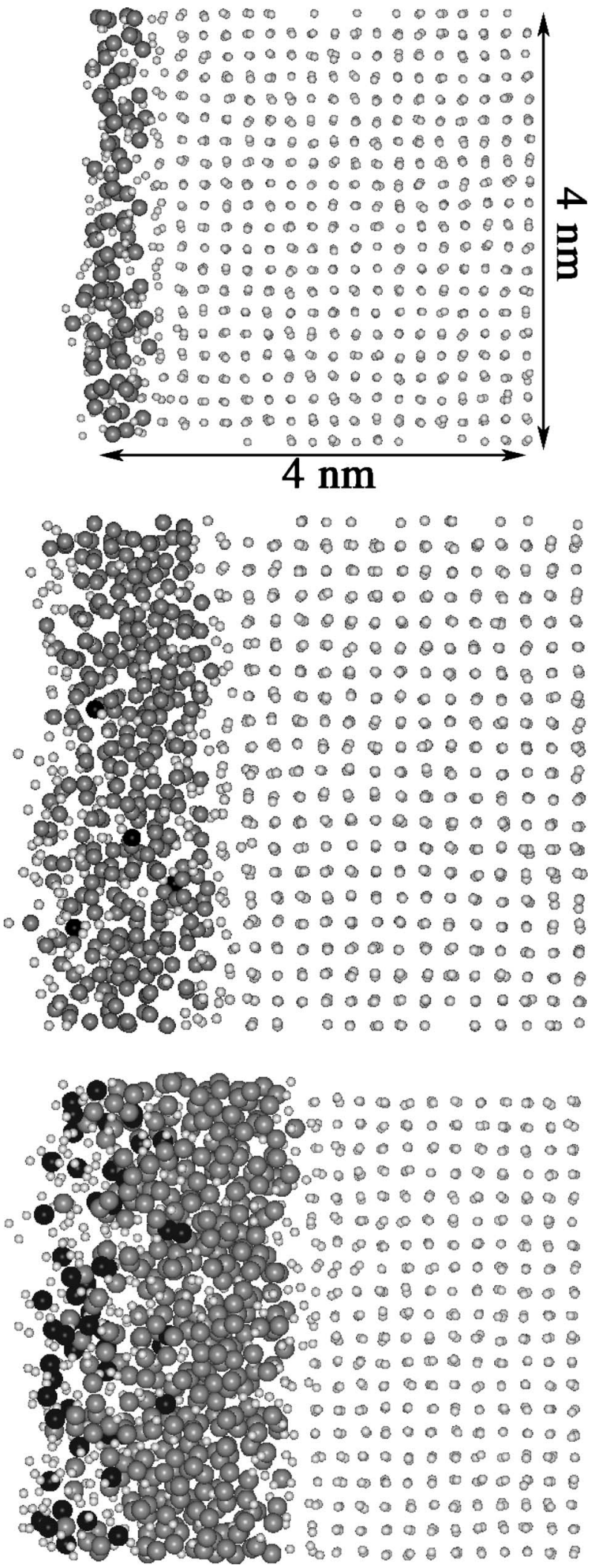

FIG. 11. A side view of a $10-\AA$-thick section of atoms showing the mixed coordination number for the (100) structure of sample B at (a) $60 \mathrm{ps}$, (b) $150 \mathrm{ps}$, and (c) $300 \mathrm{ps}$. Black balls stand for sixcoordinated atoms, dark gray for four-coordinated ones, and light gray (small) balls stand for other coordinated atoms. 
below the topmost $\mathrm{Al}$ layer (Fig. 8). Our atomistic observations, for low oxygen coverage, suggest that the oxygen adsorption occurs either within or below the geometrical surface plane (the topmost layer). This is in partial agreement with the initial stage study of Kravchuk et al. ${ }^{49}$ who suggested that the adsorption occurs below the surface plane.

Atomistic observations have shown that oxygen atoms rearrange in $(1 \times 1)$ groups or islands. In Fig. 10(a), we have shown an example of a 3 - $\AA$-thick section of atoms that show the triangular network formed by $\mathrm{Al}$ atoms in the regions containing oxygen atoms. We did not observe any oxygen single atoms as suggested by the abstractive dissociation. Moreover, the structure in Fig. 10(a) shows that individual oxygen adatoms created by disruption of some pairs will finally attach to the already formed pairs or larger groups. This process occurs during the dissociation and is not a diffusion process that takes place separately. When an oxygen molecule approaches the $\mathrm{Al}$ surface, the two oxygen atoms start to separate from each other laterally at $\sim 1 \AA$ above the Al surface. During this separation they may encounter other oxygen atoms already deposited on the surface and will attach to them, leading eventually to the formation of $(1 \times 1)$ groups by some atomic rearrangement. This explains the occurrence of groups with three or more $\mathrm{O}$ adatoms, as has been observed experimentally by Schmid et al. ${ }^{19}$ at room temperature. As mentioned in Sec. IV, the pictures in Fig. 10 are a $2 \mathrm{D}$ representation and some distortion in the perpendicular direction is not excluded. Kiejna et al. ${ }^{26}$ suggested that the most stable $\mathrm{O}-(1 \times 1)$ subsurface structure is found in tetrahedral sites below the topmost $\mathrm{Al}$ atomic plane. This is in fact an idealized picture since more detailed investigation of these sections of atoms showed that some oxygen atoms are indeed below the topmost $\mathrm{Al}$ atomic plane and others are located in the plane. The formation of oxygen $(1 \times 1)$ islands on $\mathrm{Al}$ surface at room temperature indicates that the lateral interaction between $\mathrm{O}$ atoms is attractive. However, the formation of such islands is not necessarily a result of the lateral oxygen diffusion as suggested by Jeurgens et al. ${ }^{14}$ For high values of the gas pressure as in the present case, clusters (islands) of oxygen atoms are more likely to form during the dissociation of oxygen molecules. This occurs at a distance of $0.8-1.5 \AA$ above the surface, rather than by lateral oxygen diffusion. Indeed, during the dissociation process, oxygen atoms belonging to the same molecule move away from each other when approaching the surface. In particular, when oxygen pairs (or Al-O clusters) are already present, the $\mathrm{O}$ atoms try to deposit directly on the cluster edges as suggested by Kiejna et al. ${ }^{26}$ Note also that oxygen molecules arriving on oxidized region of the Al surface do not have a vertical orientation but try rather to be horizontal when approaching the oxygen cluster (or pair) already adsorbed. This is in contrast to oxygen molecules that arrive vertically on a "clean" region of the $\mathrm{Al}$ surface.

Using fixed charge MD simulations, Gutierrez and Johansson ${ }^{34}$ have found that the coordination number of the elementary unit of the oxide increases as its density increases. Based upon this observation, they suggested that the tetrahedrally and octahedrally coordinated forms of amorphous alumina found experimentally correspond to different densities. This is in agreement with our simulation results where we found that the proportion of the $\mathrm{AlO}_{6}$ octahedron over the whole oxide becomes more important when the oxide develops and the density increases. Moreover, the contribution of the $\mathrm{AlO}_{6}$ octahedron is more important in the outer part of the oxide, which is less dense in comparison to the inner region close to the metal-oxide interface but also more rich in oxygen. In this region, $\mathrm{Al}$ atoms are then more likely to have more neighboring $\mathrm{O}$ atoms. The $\mathrm{AlO}_{4}$ tetrahedron are essential to the formation of the amorphous oxide film structure as suggested by Lamparter and Kniep. ${ }^{23}$ However, in accord with Campbell et al., ${ }^{32}$ we have observed that the $\mathrm{AlO}_{6}$ octahedron can not be neglected in the building up of the final amorphous structure. Indeed, the presence of this last oxide unit $\left(\mathrm{AlO}_{6}\right)$ is more important when the oxide develops mainly in the outer part, which is an oxygenenriched region compared to the overall amorphous oxide composition.

\section{CONCLUDING REMARKS}

We have simulated the oxidation of aluminum-single crystals with low-index surfaces [(100), (110), and (111)] between 300 and $600 \mathrm{~K}$ and under a gas pressure varying from $9.8 \times 10^{+5} \mathrm{~Pa}$ to $2.9 \times 10^{+6} \mathrm{~Pa}$. Two system sizes were used and no size effect was observed. In the studied temperature/pressure regime, the growth mechanism has been found to be independent of the crystallographic orientation. Oxide films of $\sim 3 \mathrm{~nm}$ of limiting thickness have been obtained and for films thicker than $\sim 2 \mathrm{~nm}$, the structure of the oxide presents a dominant tetrahedral environment in the inner layer and mixed tetrahedral and octahedral environments in the outer region.

The growth kinetic curves obey a direct-logarithmic law beyond a transient regime. In the theory of metal oxidation, this mode is generally associated to a growth mechanism where the oxide film develops by ion migration via mobile voids present in the amorphous oxide structure.

The temperature and gas pressure effects on the growth law have been investigated through the variation of the parameters involved in this growth law [Eq. (6)] as function of temperature and gas pressure. Our results show that the structural term $(\lambda)$ is a function of both temperature and gas pressure in contrast to what is generally accepted, i.e., a constant. The second term $\xi$ has also been found to be a temperature/gas pressure dependent term (Figs. 6 and 7).

Atomistic analysis shows that, during the dissociation stage, the oxygen molecule undergoes a rotation prior to its dissociation. The oxygen dissociation starts effectively when the center of mass of the pair is located at a position that ranges between 0.8 and $1.5 \AA$ above the surface and the final separation distance ranges from 3 to $5 \AA$, and due to atomic rearrangement and oxygen molecular interactions it may reach $9 \AA$ in some cases. During the initial stage, $\mathrm{Al}$ atoms rearrange to form a triangular network on the top layer where oxygen atoms occupy the centers of the triangles. This is correlated to the tetrahedral configuration of $\mathrm{Al}$ and oxygen atoms.

\section{ACKNOWLEDGMENTS}

We would like to thank P. Vashishta and his group for fruitful discussions, the CRI from the University of Bur- 
gundy and the CINES from Montpellier for allowing us to access their computer facilities. A. Hasnaoui and O. Politano would like to thank the Regional Consil of Burgundy and the CNRS-France for their financial support.
*Corresponding author. FAX: +33 (0)380 3961 32. Email address: olivier.politano@u-bourgogne.fr

${ }^{1}$ A. Atkinson, Rev. Mod. Phys. 57, 437 (1985).

${ }^{2}$ F. P. Fehlner, Low Temperature Oxidation (Wiley, New York, 1986).

${ }^{3}$ Corrosion of Zirconium Alloys in Nuclear Power Plants (IAEA, Vienna, 1993), Vol. 684.

${ }^{4}$ F. H. Froes, C. Suryanarayana, and D. Eleizer, J. Mater. Sci. 27, 5113 (1992).

${ }^{5}$ A. Tampieri and A. Bellosi, J. Am. Ceram. Soc. 75, 1688 (1992).

${ }^{6}$ E. McCafferty, P. M. Natishan, and G. K. Hubler, Interface (USA) 2, 45 (1993).

${ }^{7}$ J. M. De Teresa, A. Barth el emy, A. Fert, J. P. Contour, F. Montaigne, and P. Seneor, Science 286, 507 (1999).

${ }^{8}$ M. Sharma, S. X. Wang, and J. H. Nickel, Phys. Rev. Lett. 82, 616 (1999).

${ }^{9}$ T. W. Hickmott, J. Appl. Phys. 88, 2805 (2000).

${ }^{10}$ E. S. Snow, P. M. Campbell, R. W. Rendell, F. A. Buaot, D. Park, C. R. K. Marrian, and R. Magno, Semicond. Sci. Technol. 13, A75 (1998).

${ }^{11}$ A. T. M. van Gogh, S. J. van der Molen, J. W. J. Kerssemakers, N. J. Koeman, and R. Griessen, Appl. Phys. Lett. 77, 815 (2000).

${ }^{12}$ International Technology Roadmap for Semiconductors, Semiconductor Industry Association, http://public.itrs.net

${ }^{13}$ H. Lüth, Surfaces and Interfaces of Solids, Springer Series in Surface Science (Springer, Berlin, 1993).

${ }^{14}$ L. P. H. Jeurgens, W. G. Sloof, F. D. Tichelaar, and E. J. Mittemeijer, J. Appl. Phys. 92, 1649 (2002).

${ }^{15}$ L. P. H. Jeurgens, W. G. Sloof, F. D. Tichelaar, C. G. Borsboom, and E. J. Mittemeijer, Appl. Surf. Sci. 144-145, 11 (1999).

${ }^{16}$ D. Starodub, T. Gustafsson, and E. Garfunkel, Surf. Sci. 552, 199 (2004).

${ }^{17}$ O. Benka and M. Steinbatz, Surf. Sci. 525, 207 (2003).

${ }^{18}$ H. Brune, J. Wintterlin, R. J. Behm, and G. Ertl, Phys. Rev. Lett. 68, 624 (1992).

${ }^{19}$ M. Schmid, G. Leonardelli, R. Tscheliessnig, A. Biedermann, and P. Varga, Surf. Sci. 478, L355 (2001).

${ }^{20}$ L. P. H. Jeurgens, W. G. Sloof, F. D. Tichelaar, and E. J. Mittemeijer, Surf. Sci. 506, 313 (2002).

${ }^{21}$ L. P. H. Jeurgens, W. G. Sloof, F. D. Tichelaar, and E. J. Mittemeijer, Thin Solid Films 418, 89 (2002).

${ }^{22}$ L. P. H. Jeurgens, W. G. Sloof, F. D. Tichelaar, and E. J. Mitte- meijer, Phys. Rev. B 62, 4707 (2000).

${ }^{23}$ P. Lamparter and R. Kniep, Physica B 234-236, 405 (1997).

${ }^{24}$ Yu. F. Zhukovskii, P. M. W. Jacobs, and M. Causa, J. Phys. Chem. Solids 64, 1317 (2003).

${ }^{25}$ Y. Yourdshahyan, B. Razaznejad, and B. I. Lindqvist, Solid State Commun. 117, 531 (2001).

${ }^{26}$ Y. Yourdshahyan, B. Razaznejad, and B. I. Lindqvist, Phys. Rev. B 65, 075416 (2002).

${ }^{27}$ J. Jacobsen, B. Hammer, K. W. Jacobsen, and J. K. Norskov, Phys. Rev. B 52, 14954 (1995).

${ }^{28}$ T. Sasaki and T. Ohno, Surf. Sci. 433-435, 172 (1999).

${ }^{29}$ L. C. Ciacchi and M. C. Payne, Phys. Rev. Lett. 92, 176104 (2004).

${ }^{30}$ A. Kiejna and B. I. Lundqvist, Surf. Sci. 504, 1 (2002).

${ }^{31}$ S. Ogata and T. J. Campbell, J. Phys.: Condens. Matter 10, 11449 (1998).

${ }^{32}$ T. J. Campbell, R. K. Kalia, A. Nakano, and P. Vashishta, Phys. Rev. Lett. 82, 4866 (1999).

${ }^{33}$ A. Hasnaoui, O. Politano, J. M. Salazar, G. Aral, R. K. Kalia, A. Nakano, and P. Vashishta, Surf. Sci. 579, 47 (2005).

${ }^{34}$ G. Gutierrez and B. Johansson, Phys. Rev. B 65, 104202 (2002).

${ }^{35}$ F. H. Streitz and J. W. Mintmire, Phys. Rev. B 50, 11996 (1994).

${ }^{36}$ M. W. Finnis and J. E. Sinclair, Philos. Mag. A 50, 45 (1984).

${ }^{37}$ P. P. Ewald, Ann. Phys. 64, 253 (1921).

${ }^{38}$ V. A. Bakaev, Phys. Rev. B 60, 10723 (1999).

${ }^{39}$ I. C. Yeh and M. L. Berkowitz, J. Chem. Phys. 111, 3155 (1999).

${ }^{40}$ H. J. C. Berendsen, J. P. M. Postma, W. F. van Gunsteren, A. DiNola, and J. R. Haak, J. Chem. Phys. 81, 3684 (1984).

${ }^{41}$ K. Lawless, Rep. Prog. Phys. 37, 231 (1974).

${ }^{42}$ M. L. Zheludkevich, A. G. Gusakov, A. G. Voropaev, A. A. Vecher, E. N. Kozyrski, and S. A. Raspopov, Oxid. Met. 61, 39 (2004).

${ }^{43}$ N. Cabrera and N. F. Mott, Rep. Prog. Phys. 12, 163 (1949).

${ }^{44}$ H. Brune, J. Winterllin, G. Ertl, J. Wiechers, and R. J. Rehm, J. Chem. Phys. 99, 2128 (1993).

${ }^{45}$ R. Chakarova, D. E. Oner, I. Zoric, and B. Kasemo, Surf. Sci. 472, 63 (2001).

${ }^{46}$ T. Sasaki and T. Ohno, Surf. Sci. 433-435, 172 (1999).

${ }^{47}$ C. Engdahl and G. Wahnstrom, Surf. Sci. 312, 429 (1994).

${ }^{48}$ G. Wahnstrom, A. B. Lee, and J. Stromquist, J. Chem. Phys. 105, 326 (1996)

${ }^{49}$ T. Kravchuk, R. Akhvlediani, V. V. Gridin, and A. Hoffman, Surf. Sci. 562, 83 (2004). 